\title{
Immigration, neue Armut und Segregation in Peking
}

\author{
Roger Chan, Hongkong, Chaolin Gu, Nanjing, Werner \\ Breitung, Macau
}

\section{Einführung}

Im Zuge der Öffnungs- und Reformpolitik der 80er und 90er Jahre in China wurde die zuvor strikter staatlicher Kontrolle unterworfene Binnenmigration zu einem die räumliche Entwicklung Chinas prägenden Phänomen. Der Strom überschüssiger Arbeitskräfte, die auf der Suche nach neuen Möglichkeiten und bescheidenem Wohlstand in die Metropolen streben, hat enorme soziale und ökonomische Implikationen und stellt die städtischen und staatlichen Behörden vor eine Vielzahl von Problemen. Die unbeabsichtigten Folgen der Öffnungspolitik sind eine Herausforderung, die mit der Mitgliedschaft Chinas in der Welthandelsorganisation (WTO) und dem Investitionsschub für die Olympischen Spiele 2008 noch zunehmen wird. Ein Rückblick auf die auslösenden Faktoren für soziale und sozialräumliche Polarisation speziell in Peking kann helfen, diese Herausforderungen zu meistern.

Noch mangelt es an genauen Erkenntnissen über die Migrationsprozesse und insbesondere über die floating population (liudong renkou) in den Städten Chinas. Diese offiziell temporären Zuwanderer vom Lande sind polizeilich registriert, aber in den meisten amtlichen Statistiken nicht erfasst. Es gibt jedoch von Zeit zu Zeit statistische Erhebungen auf lokaler Ebene, die wertvolle Aufschlüsse geben. Dieser Artikel analysiert auf der Grundlage einer solchen Erhebung der floating population Pekings einige sozio-ökonomische Auswirkungen des durch die Landflucht verursachten Wandels in der Bevölkerungsstruktur der Stadt und leistet damit einen Beitrag zur komplexeren Migrations-, Stadt- und Sozialforschung in einem Transformations- und Entwicklungsland. Am Beispiel Pekings in den 1990er Jahren fokussiert die Analyse auf Ursachen und Merkmale städtischer Armut und städtischer Segregation.

\subsection{Chinas Urbanisierungsstrategie}

Die unter Deng Xiaoping Ende 1978 eingeleitete Öffnungs- und Reformpolitik konzentrierte sich in ihrer ersten Phase vorwiegend auf den Agrarsektor. Die Eigenverantwortung der Bauern wurde gestärkt und die Effizienz der Landwirtschaft maßgeblich gesteigert, wodurch ein Arbeitskräfteüberschuss im ländlichen Raum entstand. Reformen im städtischen Bereich wurden in der zweiten Phase, ab 1984, eingeleitet. Insbesondere im Zusammenhang mit ausländischen Direktinvestitionen entstanden in den Städten neue
Erwerbsmöglichkeiten. Es ergab sich ein Feld von Push- und Pullfaktoren, das den Strom der Landarbeiter in die Städte anwachsen ließ (BLECHER 1983, Davis 1990, Kıм 1990).

Bevölkerungswanderungen unterlagen bis 1985 der strikten Kontrolle durch ein effektives Haushaltsmeldesystem (SOLINGER 1985, Xu 1989), das zwischen städtischer und ländlicher Bevölkerung unterscheidet und den Zuzug in die Städte sehr restriktiv regelt. Dieses sogenannte hukou-System ist formal nach wie vor gültig, wird aber de facto nicht mehr durchgesetzt. Die Öffnungs- und Reformpolitik hat so indirekt und in nicht vorhergesehenem Maße Bevölkerungswanderungen innerhalb und außerhalb des Landes ermöglicht und gefördert. Die Verstädterung schreitet seitdem in alarmierendem Maße voran (Pannell 1993). 1994 gab es in China 32 Städte mit einer nichtlandwirtschaftlichen Bevölkerung von über einer Million. Mehr als 337 Millionen Menschen lebten in den großen Städten und Metropolitanregionen des Landes (Hamer 1995).

Von chinesischen Wissenschaftlern liegen Arbeiten zu Themen der Stadtentwicklung und Urbanisierungsstrategie in ihrem Land vor (Wu \& ZHANG 1984, Institute of Demographic Studies 1988, State StatiStical Bureau 1991, Chan 1994). In einer Reihe speziellerer Studien widmeten sie sich auch der sogenannten floating population und ihren Auswirkungen auf das städtische China (XU \& Li 1989, KIm 1990, MA 1990, Wu \& Xu 1990, Li 1991, Chan 1992, 1995, SHeN \& Tong 1992, Siu \& Li 1993, Qian 1996). Im deutschsprachigen Raum haben TAubManN $(1993,2000)$ und SCHARPING (1997) diese Diskussion aufgegriffen.

\subsection{Wirtschaftliche Entwicklung und soziale Spaltung} Jenseits der Situation in China geben MingIONE (1987) und Todaro (1989) einen Überblick über traditionelle Sichtweisen zu Urbanisierung und städtischem Wandel. Oshima (1987) und McGee (1991) behandelten die Urbanisierung in Asien als eine Entwicklungsstrategie. Clinard \& Aвbot (1973) untersuchten das Wachstum der Primatstadt, die «...wegen ihrer zunehmenden Austrahlung, Modernität und Vielschichtigkeit eine wachsende Anzahl von Zuwanderern anzieht». Sumner (1982) wies dann auch auf die mit der Industrialisierung und Urbanisierung verbundenen «Asymmetrien» hin:zunehmende Arbeitslosigkeit, Mangel an ausgebildeten Arbeitskräften, wirtschaftlicher Dualismus und Abnahme familiärer Werte. Er argumentierte, dass eine Industrialisierung Entwicklungsländern aufgrund solcher mit dem Streben nach Modernität verbundenen Erscheinungen auch zum Schaden gereichen kann. 
Der Prozess der Migration in die Städte in China unterscheidet sich nicht grundsätzlich von dem in der übrigen Dritten Welt. Die Konzentration ausländischer Direktinvestitionen auf die Städte schafft dort eine kleine aber wohlhabende Schicht Neureicher und einen wachsenden Zustrom Armer auf der Suche nach sozialen Aufstiegsmöglichkeiten. Die rasche Zunahme der floating population in den Metropolitanregionen führte dort zu steigender Arbeitslosigkeit. Besonders vor dem Hintergrund der gleichzeitigen Umstrukturierung in den Staatsbetrieben Chinas nimmt die Zahl der überschüssigen Arbeitskräfte zu, und eine neue soziale Gruppe der Arbeitslosen wächst heran. Die Schere zwischen den hohen und den zum Teil sehr niedrigen Einkommen öffnet sich zunehmend. Es gibt einen direkten Zusammenhang zwischen den wirtschaftlichen Entwicklungsprozessen und diesen Trends in China (DAvis et al. 1995).

\subsection{Neue Armut in den Städten}

Die Diskussion um die neue Armut und wachsende Disparitäten in den Städten des Westens berücksichtigt nicht die Situation in den Städten Chinas, was bislang insofern gerechtfertigt schien, als letztere nur geringe soziale Disparitäten aufwiesen. Inzwischen ändert sich das aber dramatisch, und durch die Möglichkeit zur Abwanderung in die Städte schrumpft auch die räumliche Distanz zwischen Armut und Reichtum. Viele z.B. der folgenden von Room (1990) aufgeführten Merkmale einer neuen Armut in den Städten des Westens sind inzwischen in Peking und anderen chinesischen Städten ebenso feststellbar:

- steigende Zahl von Menschen, die auf Sozialleistungen angewiesen sind

- steigende Zahl Alleinerziehender
- steigende Arbeitslosigkeit und Zeitarbeitsverträge auch in Berufsfeldern der Mittelklasse

- zunehmende Verschuldung von Menschen unterhalb der Armutsgrenze

- zunehmende Obdachlosigkeit.

Wie in den Städten des Westens ist diese Entwicklung zum Teil auf einen Rückgang staatlicher Regulation und auf Auswirkungen der Globalisierung zurückzuführen. Auch in westlichen Städten wird ein Großteil der prekären Arbeitsverhältnisse von Immigranten eingenommen, deren unsicherer Aufenthaltsstatus dabei ausgenutzt wird. Es gibt allerdings auch ganz wesentliche Unterschiede. Die floating population der chinesischen Städte hält überwiegend enge Kontakte in die ländlichen Regionen aufrecht. Eine Rückkehr ist jederzeit möglich, und der Aufenthalt in der Stadt aus der Sicht des Individuums trotz der fatalen Situation dort keine Sackgasse sondern eine aktiv betriebene Aufstiegsstrategie.

\section{Sozialstruktur der floating population in Peking}

Auf der Grundlage der Erhebung der floating population durch die Pekinger Stadtregierung im November 1994 (Beising Evening News 1995), untersucht das folgende Kapitel die Ursachen der neuen Armut dort und berücksichtigt dabei Erwerbsstruktur, Familiensituation, Alter, Geschlecht, Bildungsniveau und Arbeitsteilung. Es zeigt sich, dass floating population ein sehr heterogener Begriff ist, der neben der großen Gruppe der Wanderarbeiter auch Geschäftsleute, Akademiker, Touristen etc. ohne offizielle städtische Wohnberechtigung umfasst.

\begin{tabular}{|l|r|}
\hline Kleingewerbe, Gastronomie und Kleinhandel & $34,9 \%$ \\
\hline Bauwesen & $21,4 \%$ \\
\hline Fabrikarbeit & $5,0 \%$ \\
\hline Haushaltshilfe & $1,9 \%$ \\
\hline Landwirtschaft & $0,9 \%$ \\
\hline Geschäftsreisende & $12,5 \%$ \\
\hline Bildung und Ausbildung & $4,0 \%$ \\
\hline Touristen & $2,3 \%$ \\
\hline Krankenhausaufenthalt & $1,7 \%$ \\
\hline Sonstige & $15,4 \%$ \\
\hline Gesamt & $100,0 \%$ \\
\hline
\end{tabular}

Tab. 1: Floating population in Peking nach Tätigkeitsfeld (1994)

Floating population in Beijing according to occupation (1994)

Population migrante interne à Pekin, selon son activité (1994)

Quelle: Beising Evening News 17.6.1995: 3 


\begin{tabular}{|r|r|l|}
\hline \multicolumn{1}{|c|}{ Alter } & \multicolumn{1}{|c|}{$\%$} & typische Lebenssituation \\
\hline unter 1 & 1,0 & in Peking neu geboren \\
\hline $1-5$ & 5,3 & bei den Eltern lebend \\
\hline $6-14$ & 3,6 & $45 \%$ besuchen eine Schule, 10 \% arbeiten (ab 10 Jahre alt) \\
\hline $15-44$ & 80,0 & überwiegend Arbeiter \\
\hline $45-59$ & 7,9 & Geschäftsleute oder in Forschung, Technologie und Informationswesen \\
\hline über 59 & 2,2 & Ruheständler, Nachzug zur Familie \\
\hline
\end{tabular}

Tab. 2: Altersstruktur der floating population in Peking (1994)

Age structure of floating population in Beijing (1994)

Structures d'âge de la population migrante interne à Pekin (1994)

Quelle: BEIJING EVENING News 19./20.6.1995: 3

\subsection{Schwerpunkt im Niedriglohnbereich}

Die floating population Pekings besteht mehrheitlich aus Bauern aus der Provinz. Sie arbeiten in verschiedenen Gewerben und Tätigkeiten (Tabelle 1), die überwiegend als gefährlich, schmutzig und körperlich anstrengend gelten. Hinsichtlich der Arbeitsbeziehungen lassen sich folgende vier Gruppen unterscheiden:

(1) Arbeitsintensive, schlecht bezahlte aber formelle Tätigkeiten. Obwohl der größte Teil der floating population im nicht-landwirtschaftlichen Bereich arbeitet, liegt sein Lohnniveau wesentlich niedriger als das der formellen Stadtbewohner. Die meisten Zuwanderer haben einheimische Bau- und Fabrikarbeiter ersetzt, verdienen aber nur ein Drittel bis halb so hohe Löhne wie diese.

(2) Zeitlich befristete und kurzfristige Tätigkeiten. Einige Zuwanderer arbeiten zeitlich befristet als Haushaltshilfen, Babysitter, Möbelflicker, Boten, Straßenkehrer usw.

(3) Unsichere Gelegenheitsarbeiten. Andere gehen Gelegenheitsarbeiten als Händler, Hausierer, Messerschleifer oder Rikschafahrer nach, reparieren Schuhe, Fahrräder, Uhren, fertigen zum Beispiel Stempel an oder transportieren Müll ab.
(4) Selbständige Tätigkeiten. In jüngster Zeit gibt es darüber hinaus auch einige wirtschaftlich erfolgreiche Gründungen kleiner Firmen, Restaurants und Fabriken durch Zuwanderer vom Lande. Ein gutes Beispiel in Peking ist das sogenannte "Zhejiang-Viertel» von Zuwanderern aus dieser Küstenprovinz, die im wesentlichen in der Bekleidungsindustrie tätig sind. Ihre meist als Familienbetriebe geführten Nähwerkstätten breiteten sich rasch über das Viertel aus. Ein anderes Beispiel für ein erfolgreiches durch Zuwanderer gegründetes Unternehmen in Peking ist das von Migranten aus Guangdong geführte kantonesische New-Ajing-Restaurant.

\subsection{Alter und Lebenssituation}

Nach der Erhebung von 1994 lag das Durchschnittsalter der floating population bei 27,7 Jahren (Männer: 28,5, Frauen: 26,4). Die Zuwanderer sind damit im Schnitt 6,6 Jahre jünger als die ansässige Bevölkerung. Generell ist die floating population jünger und auf dem Arbeitsmarkt konkurrenzfähiger als die ansässigen Pekinger Arbeitskräfte. Allerdings können nur $45 \%$ ihrer Kinder eine Schule besuchen (Tabelle

\begin{tabular}{|l|r|r|}
\hline Bildungsstand & der Zuwanderer gesamt & \multicolumn{2}{|c|}{ der Zuwanderinnen } \\
\hline Hochschule & $3,1 \%$ & $2,5 \%$ \\
\hline Oberschule & $11,7 \%$ & $8,6 \%$ \\
\hline Mittelschule & $54,8 \%$ & $24,3 \%$ \\
\hline Grundschule & $24,9 \%$ & $51,0 \%$ \\
\hline Analphabeten & $5,5 \%$ & $13,6 \%$ \\
\hline
\end{tabular}

Tab. 3: Floating population in Peking nach Bildungsstand (1994)

Floating population in Beijing according to level of education (1994)

La population migrante interne à Pekin, selon son niveau de formation (1994)

Quelle: BeiJing Evening News 19./20.6.1995: 3 


\begin{tabular}{|l|r|l|}
\hline Familienstand & \multicolumn{1}{|c|}{ Anteil } & durchschnittliche Kinderzahl \\
\hline ledig & $36,8 \%$ & - \\
\hline verheiratet & $60,9 \%$ & 1,3 Kinder (28,5\% mit 2 Kindern, 8,1\% mit 3 Kindern) \\
\hline verwittwet & $2,1 \%$ & 1,3 Kinder \\
\hline geschieden & $0,2 \%$ & 1,3 Kinder \\
\hline
\end{tabular}

Tab. 4: Zuwanderinnen in Peking nach Familienstand (1994)

Female immigrants in Beijing according to marital status (1994)

Immigrées à Pékin, selon la situation familiale (1994)

Quelle: BeiJing Evening News 19.6.1995: 3

2). Die floating population aus den ländlichen Regionen hat im Gegensatz zu den Bürgern der Städte keinen Anspruch auf städtische Leistungen wie Erziehungs-, Gesundheitseinrichtungen und Versorgung mit Wohnraum. Auch der Zugang zu Arbeit in Staatsbetrieben und sogar in ausländischen bzw. joint-venture-Unternehmen ist für sie normalerweise nicht einfach.

\subsection{Niedriges Bildungsniveau}

Durch den Strukturwandel der chinesischen Städte von Industrie zu Dienstleistungen, Finanzwirtschaft und High-Tech-Sektor, werden zunehmend Arbeitskräfte mit höherem Ausbildungsstand benötigt. Nur die wenigsten der neuen Zuwanderer hatten aber eine Hochschulausbildung, und immerhin 5,5\% waren Analphabeten oder kaum alphabetisiert. Die besser
Ausgebildeten arbeiten meist in Staatsbetrieben oder der öffentlichen Verwaltung. Die Erhebung zeigte auch, dass das Ausbildungsniveau der Zuwanderinnen niedriger war als das der männlichen Zuwanderer (Tabelle 3). In Abhängigkeit von dem Ausbildungsniveau stehen der Mehrheit der floating population nur unsichere und schlecht bezahlte Arbeitsplätze mit niedrigem sozialen Status offen. Nur eine Minderheit hat Chancen auf eine feste Anstellung mit guter Bezahlung.

\subsection{Geringer Frauenanteil}

Die floating population Pekings ist überwiegend männlich. Die Erhebung 1994 ergab einen Männeranteil von $63,4 \%$, was darauf zurückgeführt werden kann, dass im Niedriglohnsektor physische und schmutzige Arbeiten, z. B. auf Baustellen und in Fabriken

\begin{tabular}{|l|l|l|}
\hline Provinz & männliche Zuwanderer & weibliche Zuwanderinnen \\
\hline Hebei & Bauwesen, Geschäfte & Geschäfte, Gastronomie, Haushaltshilfen \\
\hline Zhejiang & $\begin{array}{l}\text { Geschäfte, Herstellung von Kleidung, } \\
\text { Schuhen, Reparaturen }\end{array}$ & $\begin{array}{l}\text { Geschäfte, Herstellung von Kleidung, } \\
\text { Schuhen, Reparaturen }\end{array}$ \\
\hline Henan & Bauwesen, Abfallsammlung, Geschäfte & Geschäfte, Gastronomie, Haushaltshilfen \\
\hline Anhui & Bauwesen, Geschäfte, Industrie & Haushaltshilfen \\
\hline Sichuan & Bauwesen, Geschäfte, Gastronomie & $\begin{array}{l}\text { Gastronomie, Haushaltshilfen, Hotellerie, } \\
\text { Krankenpflege }\end{array}$ \\
\hline Jiangsu & Bauwesen, Reparaturen & $\begin{array}{l}\text { Reparaturen, Kleingewerbe oder Klein- } \\
\text { handel }\end{array}$ \\
\hline Shandong & Bauwesen, Kleingewerbe & Reparaturen, Kleingewerbe, Kleinhandel \\
\hline andere & $\begin{array}{l}\text { Bauwesen, Kleingewerbe, } \\
\text { Industrie, Transportwesen, } \\
\text { Gastronomie }\end{array}$ & Kleingewerbe, Industrie, Gastronomie \\
\hline
\end{tabular}

Tab. 5: Hauptsächliche Tätigkeitsfelder der Zuwanderer nach Herkunftsprovinzen (1994)

Main occupations of immigrants according to provinces of origin (1994)

Principaux secteurs d'activité des immigrés, selon la province d'origine (1994)

Quelle: Beijing Evening News 19.6.1995: 3 
überwiegen. Die Zuwanderinnen arbeiteten vor allem in schlecht bezahlten Dienstleistungsberufen. 59,8\% von ihnen waren im Alter von 15-29 Jahren. Die meisten waren verheiratet und hatten im Durchschnitt 1,3 Kinder (Tabelle 4). Überwiegend waren sie allerdings alleinerziehend. Sie brauchten ihren Verdienst um die Familie zu ernähren, während die Zuwanderer sonst vor allem Ersparnisse für Hochzeiten und das Bauen oder Erneuern von Häusern in den jeweiligen Heimatorten anstrebten.

\subsection{Unterschiede nach Herkunftsregion}

Es fällt ein enger Zusammenhang zwischen Geschlecht und Herkunftsregion der Migranten und ihren Tätigkeiten in Peking auf. Die Erhebung zeigte, dass männliche Zuwanderer aus den Provinzen Jiangsu, Shandong und Hebei schwerpunktmäßig im Bauwesen arbeiten, z.B. als Schreiner, Maurer oder Klempner, während viele aus Henan sich ihren Lebensunterhalt als Abfallsammler, Straßenreiniger oder Gießer verdienen und einige aus Shandong z.B. als Gemüsehändler. Zuwanderinnen aus Jiangsu sind oft als Geschäftsfrauen, Spinnerinnen oder Fabrikarbeiterinnen tätig, diejenigen aus Sichuan und Nordostchina zum großen Teil in Restaurants, Hotels und verwandten Bereichen. Zuwanderer aus Xinjiang und Ninxia betreiben vielfach muslimische Restaurants, und solche aus Tibet oder Qinghai handeln mit chinesischen Arzneien und Heilkräutern (Tabelle 5).

\section{Zuwanderung und soziale Segregation}

In Forschungen zu außerchinesischen Städten wurde gezeigt, dass eine soziale Polarisierung in den Städten über den Wohnungsmarkt zu stadträumlicher Segregation geführt hat (ForRest \& MURIE 1988, TAN 1993, Kempen 1994, Lash \& Urry 1994). Slums, Ghettos und soziale Segregation, die in China bisher als gesellschaftliche Fehlentwicklungen des Westens angesehen wurden, werden nun auch in chinesischen Städten zu drängenden Problemen. Die armen Zuwanderer aus den ländlichen Regionen auf der einen und der schnelle Reichtum der neuen Unternehmer auf der anderen Seite führen durch soziale Segregation in jüngster Zeit zu gravierenden sozialen und sozialräumlichen Spaltungen.

\subsection{Ungleichmäßige räumliche Verteilung der Stadtbewohner}

Tabelle 6 zeigt eine sehr ungleichmäßige räumliche Verteilung der Bevölkerung Pekings. Die Gebiete mit hoher Bevölkerungsdichte konzentrierten sich auf vier Innenstadtbezirke von zusammen nur $87 \mathrm{~km}^{2}$, nämlich der Weststadt, der Oststadt, Xuanwu und Chongwen. Das Chunshu-Quartier in Xuanwu und das Qianmen-Quartier in Chongwen waren die zwei Quartiere (chinesisch: jiedao - eine Kategorie der städtischen Verwaltungshierarchie) mit den höchsten Bevölkerungsdichten (48.182 bzw. 44.037 Einwohner pro $\mathrm{km}^{2}$ ). Drei der inneren Vorortbezirke (Shijingshan, Fengtai und Haidian) registrierten Bevölkerungsdichten von jeweils unter 10.000 Einwohnern pro $\mathrm{km}^{2}$. Die Werte außerhalb der Dritten Ringstraße lagen generell unter 5.000 Einwohner pro $\mathrm{km}^{2}$. Das LaozhuangziQuartier in Fengtai, das Sijiqing-Quartier in Haidian und das Huangguang-Quartier in Chaoyang wiesen mit 89,152 bzw. 270 Einwohner pro $\mathrm{km}^{2}$ die geringsten Bevölkerungsdichten auf (Beijing Statistical Yearвоок 1995). Dieses Gefälle der Bevölkerungsdichte

\begin{tabular}{|l|r|r|r|r|r|r|r|r|r|r|r|}
\hline $\begin{array}{l}\text { Bevölkerungs- } \\
\text { dichte in } 1000 \\
\text { Pers. pro km }\end{array}$ & $\begin{array}{l}\text { über } \\
40\end{array}$ & $\begin{array}{l}35- \\
40\end{array}$ & $\begin{array}{l}30- \\
35\end{array}$ & $\begin{array}{l}20- \\
30\end{array}$ & $\begin{array}{l}10- \\
20\end{array}$ & $\begin{array}{l}5- \\
10\end{array}$ & $3-5$ & $1-3$ & $\begin{array}{l}0,5- \\
1\end{array}$ & $\begin{array}{l}\text { unter } \\
0,5\end{array}$ & $\begin{array}{l}\text { Quartiere } \\
\text { gesamt }\end{array}$ \\
\hline Xuanwu & 2 & 2 & 1 & 2 & 1 & - & - & - & - & - & 8 \\
\hline Chongwen & 2 & - & 1 & 2 & 2 & - & - & - & - & - & 7 \\
\hline Oststadt & - & 1 & 3 & 5 & 1 & - & - & - & - & - & 10 \\
\hline Weststadt & - & - & 2 & 3 & 4 & - & - & - & - & - & 10 \\
\hline Chaoyang & - & 1 & - & 4 & 6 & 3 & 4 & 14 & 12 & 2 & 46 \\
\hline Haidian & - & - & - & - & 9 & 3 & 1 & 2 & 5 & 7 & 27 \\
\hline Fengtai & - & - & - & 1 & - & 2 & 2 & 5 & 3 & 2 & 15 \\
\hline Shijingshan & - & - & - & - & 1 & 3 & 3 & 2 & 1 & - & 10 \\
\hline gesamt & 4 & 5 & - & 17 & 24 & 11 & 10 & 23 & 21 & 11 & 133 \\
\hline
\end{tabular}

Tab. 6: Bevölkerungsdichte in Peking nach Bezirken 1993 (jeweils Zahl der Quartiere)

Population density in Beijing according to districts 1993 (number of quarters)

Densité de population de Pékin, selon les districts (1993), respectivement le nombre des quartiers

Quelle: Beijing Statistical Yearbook 1995 


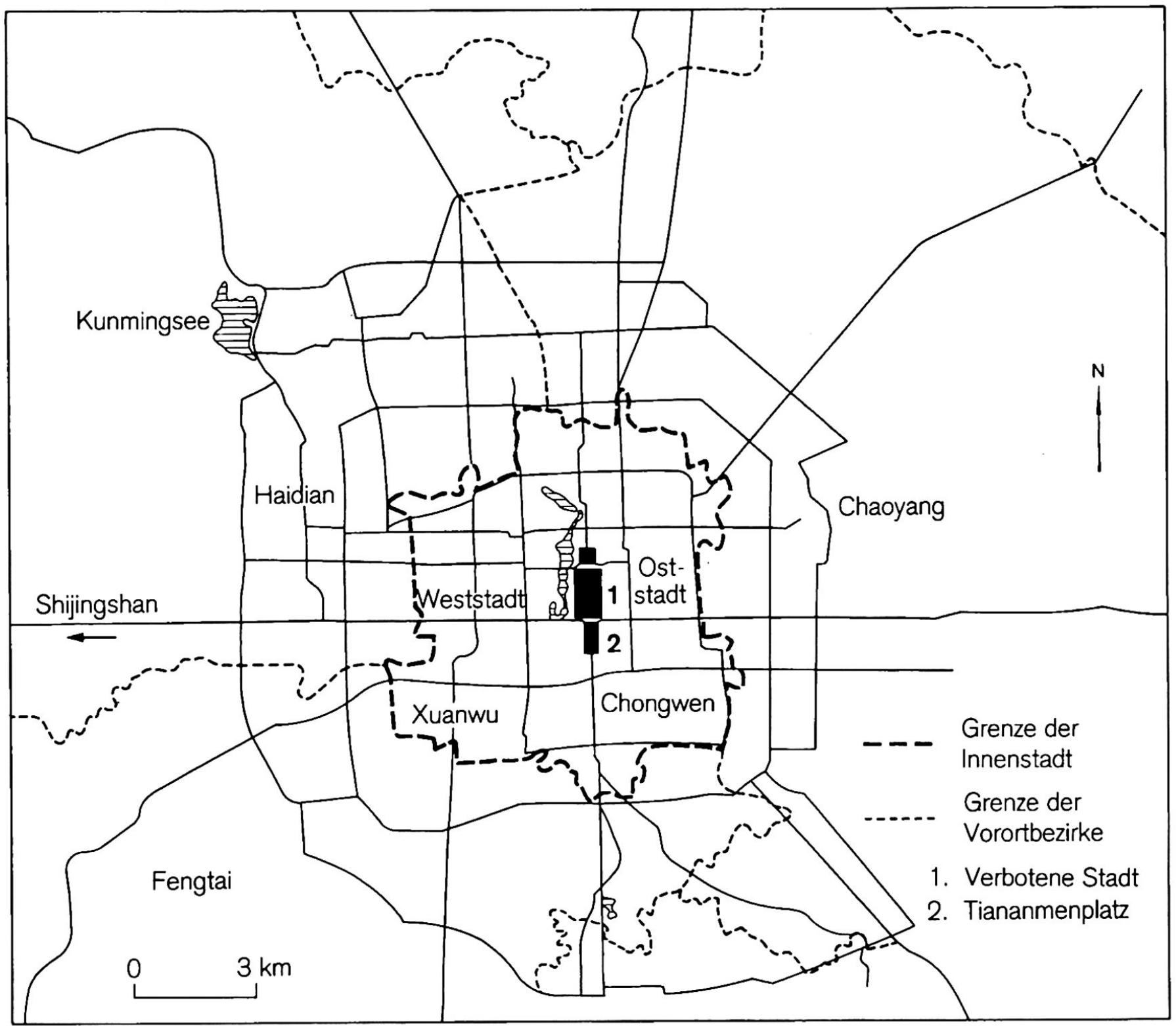

Karte 1: Innenstadt- und Vorortbezirke von Peking Inner urban and inner suburb districts of Beijing Districts du centre-ville et de la périphérie de Pékin Kartographie: L. BAUMANN

mit der Entfernung ist der Bezugsrahmen, mit dem die sozialräumliche Verteilung der floating population überprüft werden muss.

\subsection{Räumliche Verteilung der floating population}

Die Pekinger Erhebung zur floating population beziffert die Zahl dieser temporären Neuzuwanderer mit 2,877 Millionen, gibt aber kaum Hinweise auf ihre räumliche Verteilung. Nach dem Statistischen Jahrbuch Pekings von 1995, das allerdings nur eine floating population von 875.000 erfasst, lebten $66,6 \%$ von ihnen in den drei inneren Vorortbezirken Chaoyang, Haidian und Fengtai (Tabelle 7). Dadurch stieg das zahlenmäßige Verhältnis von ihnen zu den offiziellen Einwohnern dort auf 1:6. Die Oststadt und Xuanwu waren Bezirke mit einer hohen Dichte der floating population. Fengtai und Chaoyang wiesen einen besonders hohen Anteil von Zuwanderern an der Bevölkerung auf. Die höchste absolute Zahl konzentrierte sich in Chaoyang, das über die letzten Jahre ein starkes Wirtschaftswachstum erlebte und ein bevorzugter Ort für Erstzuzüge war. 


\begin{tabular}{|l|r|r|r|r|r|r|}
\hline & $\begin{array}{l}\text { Gesamt- } \\
\text { bevölkerung } \\
\text { in } 10.000\end{array}$ & $\begin{array}{l}\text { offizielle } \\
\text { Einwohner } \\
\text { in } 10.000\end{array}$ & $\begin{array}{l}\text { floating } \\
\text { population } \\
\text { in } 10.000\end{array}$ & $\begin{array}{l}\text { Fläche } \\
\text { in km }\end{array}$ & $\begin{array}{l}\text { Verhältnis floating } \\
\text { population zu offi- } \\
\text { ziellen Einwohnern }\end{array}$ & $\begin{array}{l}\text { floating } \\
\text { population in } \\
\text { Pers. pro km }\end{array}$ \\
\hline INNENSTADT & 266,5 & 243,6 & 22,9 & 87,1 & 0,09 & 2.629 \\
\hline - Weststadt & 85,8 & 78,9 & 6,9 & 30,00 & 0,09 & 2.300 \\
\hline - Oststadt & 72,0 & 64,3 & 7,7 & 24,70 & 0,12 & 3.117 \\
\hline - Xuanwu & 62,2 & 57,1 & 5,1 & 16,50 & 0,09 & 3.091 \\
\hline - Chongwen & 46,5 & 43,3 & 3,2 & 15,90 & 0,07 & 2.013 \\
\hline INNERE & 441,9 & 381,5 & 60,4 & 1282,8 & & \\
VORORTE & 157,5 & 140,3 & 17,2 & 426,00 & 0,16 & 471 \\
\hline - Haidian & 159,5 & 136,1 & 23,4 & 470,80 & 0,12 & 404 \\
\hline - Chaoyang & 89,1 & 74,2 & 14,9 & 304,20 & 0,17 & 479 \\
\hline - Fengtai & 35,8 & 30,9 & 4,9 & 81,80 & 0,20 & 489 \\
\hline - Shijingshan & 708,4 & 625,1 & 83,3 & 1369,90 & 0,16 & 599 \\
\hline GESAMT & & & & & 0,13 & 608 \\
\hline
\end{tabular}

Tab. 7: Räumliche Verteilung der floating population in Peking (1994)

Spatial distribution of floating population of Beijing (1994)

Répartition spatiale de la population migrante interne de Pékin (1994)

Quelle: BeiJing STatistical Yearbook 1995

\subsection{Segregation unter den verschiedenen Gruppen der floating population}

Bei den Zuwanderern in Peking ist ein enger Zusammenhang zwischen Arbeitsstätten und Wohnorten feststellbar. Nach der Erhebung der floating population (Beising Evening News 16.6.1995: 3) wohnten 84\% der Bauarbeiter auf den jeweiligen Baustellen (469.000 Personen). Sie stehen am unteren Ende der sozialen Skala. Durch die räumliche Konzentration dieser Bauarbeiter sind am Stadtrand regelrechte Slumgebiete entstanden. Auch über die Gruppe der Bauarbeiter hinaus lassen sich die neuen Zuwanderer vom Lande generell bevorzugt am Stadtrand nieder, wo sie billige Zimmer finden können (Gu 1995). 328.000 Zuwanderer leben außerhalb der Stadt bei Familien zur Untermiete oder in einer landwirtschaftlichen Einheit. Von den Kleinunternehmern und Beschäftigten des Dienstleistungssektors unter den Zuwanderern (577.000 Personen) lebten allerdings etwa $80 \%$ in der Innenstadt. Diejenigen von ihnen, die für joint ventures und ausländische Firmen arbeiteten (insgesamt 44.000 Personen), wohnten mitunter in Vierund Fünfsternehotels, andere in Luxuswohnungen ihrer Unternehmen. Erfolgreiche Jungunternehmer und selbständige Geschäftsleute haben in den Vororten vor allem des Nordens und Nordostens ihre Villen errichtet. Die Daten der Erhebung zeigen, dass etwa drei Fünftel $(61,16 \%)$ der nouveau riche in den Bezir- ken Chaoyang und Haidian wohnte (Chengshi Jiaoqu) und etwa ein Fünftel $(19,88 \%)$ im Bezirk Oststadt (Chengshi Shiqu).

Die Segregation ist also eine doppelte. Einerseits ist der Anteil der Zuwanderer in den Vororten (15,8 \% der Bevölkerung) wesentlich höher als in der Innenstadt $(8,6 \%)$, und andererseits gibt es eine Polarisierung zwischen den südlichen und den nördlichen Vororten. Ersteres liegt im System der städtischen Wohnraumversorgung in China begründet. Das Prinzip möglichst niedriger Mieten, und damit geringer Mittel für Neubau, Sanierung und Instandhaltung von Wohnbauten hat die städtische Wohnungspolitik in eine ernste Notlage gebracht. Das förderte die Bildung eines Schwarzmarktes, auf dem die Mieten für staatliche Wohnungen höher sind als die für private. Privater Wohnraum in der Stadt selbst ist wiederum teurer als in der ländlichen Umgebung. Hinzu kommt, dass die Vermietung der städtischen Wohnungen oft durch die Betriebe erfolgt. Aus diesen Gründen haben Zuwanderer meist keine andere Wahl als Behelfsunterkünfte entweder im Außenbereich der Stadt oder in der alten Innenstadt zu finden. Die Konzentration von gut bezahlten Zuwanderern, vor allem im Zusammenhang mit ausländischen Firmen, im Norden und Nordosten der Stadt erklärt sich mit der Lage des internationalen Flughafens im Nordosten und mit dem Quartier im Norden, das zu den Asienspielen 1990 mit gut ausge- 
bauter Infrastruktur versehen wurde. Obwohl vielfach gehofft wurde, dass die Ausrichtung der Olympischen Spiele genutzt wird, um einen Entwicklungsimpuls im armen (südlichen) Bezirk Fengtai zu setzen, entschied man sich für die risikoärmere Variante, den vormaligen Standort der Asienspiele. Es ist daher zu erwarten, dass die Investitionen im Zusammenhang mit den Spielen 2008 eher zu einer Verstärkung der sozialräumlichen Disparitäten beitragen werden.

\section{Schlussfolgerungen}

Die chinesische Stadtplanung nach 1949 hatte sich zum Ziel gesetzt, die Übel des überwundenen Regimes zu beseitigen und an der klassenlosen Gesellschaft mitzubauen. Diese politischen Ziele waren als die «drei Dienste» (sanfuwu) bekannt (MA 1979). In der gesamten Mao-Ära wurde die Idee einer Transformation von Konsumentenstädten in Produzentenstädte verfolgt und umgesetzt. Die Entwicklung in der Zeit seit Maos Tod weist dann aber auf einen deutlichen Kurswandel hin. Städtische und ländliche Räume entwickeln sich in dieser Zeit auseinander. Wie in diesem Artikel dargelegt wurde, stellt die daraus folgende zunehmende Binnenmigration eine große Belastung für die chinesische Gesellschaft und ihr Wirtschaftssystem dar. Die Diskrepanzen zwischen Stadt und Land und zwischen den Gewinnern und Verlierern in der Gesellschaft nehmen zu. Ihnen muss politisch entgegengewirkt werden. Allerdings scheint es heute ausgeschlossen, zu der früheren strikten Reglementierung zurückzukehren. Im Gegenteil, es gibt Bestrebungen, das hukou-System zumindest für Städte mittlerer Größe in absehbarer Zeit ganz aufzugeben. Dies böte die Möglichkeit, Rechtsunsicherheit und Chancenungleichheiten abzubauen. Andererseits würden damit aber die Migrationsströme weiter zunehmen, was das Land vor immense planerische und städtebauliche Aufgaben stellen würde. Regionale Disparitäten zu verringern und die ländliche Entwicklung zu fördern, scheint somit dringend geboten, um die Abwanderung von Arbeitskräften aus den ländlichen Regionen einzudämmen. Ein neues Denken im Angehen der Probleme im Zusammenhang mit der floating population kann in der Forschung zur Bevölkerungspolitik sowohl Chinas als auch anderer agrarisch geprägter Gesellschaften zu neuen Paradigmen führen.

\section{Literatur}

Beijing Evening News (1995): Artikelserie über die Erhebung der floating population Pekings. - In: Beijing Wan Bao, 16.-20.6.1995. Peking: 3 (in Chinesisch). Beijing Statistical Yearbook (1995). - Peking: China Statistical Publishing House.

BLECHER, M. (1983): Peasant labor for urban industry: temporary contract labor, urban-rural balance and class relations in a Chinese county. - In: World Development 11/8: 731-745.

CHAN, K.W. (1994): Cities with invisible walls: reinterpreting urbanization in post-1949 China. - Hongkong: Oxford University Press.

CHAN, R.C.K. (1992): Challenges to urban areas: The floating population. - In: Kuan H.C. \& M. Brosseau (Hrsg.): China Review 1992. - Hongkong.

CHAN, R.C.K. (1995): The urban migrants: the challenge to public policy. - In: Wong, L. \& S. MACPHERSON (Hrsg.): Social change and social policy in contemporary China. - London: Avebury.

Clinard, M. \& D. Аввотt (1973): Crime in developing countries. - New York: Wiley.

Davis, D. (1990): Urban job mobility. - In: Davis, D. \& E. Vogel (Hrsg.): Chinese society on the eve of Tiananmen: The impact of reform. - Harvard: 85-108.

DAvis, D. et al. (1995): Urban spaces in contemporary China: the potential for autonomy and community in post-Mao China. - Woodrow Wilson Centre Press/ Cambridge University Press.

Forrest, R. \& A. Murie (1988): Selling the welfare state: the privatization of public housing. - London: Routledge.

Gu, C. (1995): China's urban housing system in transition. - In: Journal of Chinese Geography 6, 2: 16-38.

Hamer, A. (1995): Urban China: looking forward, looking back. - In: YEH, A.G.O. \& C.K. MAK (Hrsg.): China's cities and China's development: A preview of the future role of Hong Kong. - Centre of Urban Planning and Environmental Management, University of Hong Kong.

Institute of Demographic Studies, Chinese Academy of Social Sciences (1988): Zhongguo Renkuo Qianyi yu Chengshihua Yanju (Studien zur Bevölkerungswanderung und Urbanisierung in China). - Peking: Institute of Economic Press (in Chinesisch).

Kempen, E.T. (1994): The dual city and the poor: social polarisation, social segregation and life chances. - In: Urban Studies 31, 7: 995-1015.

KIM, W.B. (1990): Population distribution policy in China: A review. - In: Regional Development Dialogue 11, 1: 159-87.

LASH, S. \& J. URRY (1994): Economies of signs and space. - London: Sage Publication.

LI, M.B. (Hrsg.) (1991): Liudong Renkou Dui Da Chengshi Fazhan De Yingxiang Yi Duice (Floating population und Großstadtentwicklung: Auswirkungen und Folgerungen). - Economic Daily Press, Beijing (in Chinesisch).

MA, L.J.C. (1979): The Chinese approach to city planning: policy, administration, and action. - In: Asian Survey 19, 9: 838-855.

MA, X. (1990): Two types of shifts in rural population. - In: Beijing Review 33, 18: 14-16.

McGeE, T. (1991): The emergence of Desakota regions 
in Asia: expanding a hypothesis. - In: GINSBURG, N. (Hrsg.): The extended metropolis: settlement transition in Asia. - Honolulu: 3-25.

Mingione, E. (1987): The urban question in socialist developing countries. - In: D. Forbes \& N. ThrifT (Hrsg.): The socialist Third World. - Oxford: 27-52.

Oshima, H. (1987): The transition from an agricultural to an industrial economy in East Asia. - In: Economic Development and Cultural Change 34: 784-809.

PANnell, C. (1993): A preface to China's urbanization: the role of large cities. - Vortrag im Centre of Urban Planning and Environmental Management der University of Hong Kong.

QIAN, W.B. (1996): Rural-urban migration and its impact on economic development in China. - London: Avebury.

Room, G. (1990): New poverty in the European Community. - London: Macmillan.

SchARPING, T. (Hrsg.) (1997): Floating population and migration in China. = Mitteilungen des Instituts für Asienkunde 284, Hamburg.

SHEN, Y.M. \& C.Z. Tong (1992): Zhongguo Renkou Qianyi (Bevölkerungswanderungen in China).-Peking: Chinese Statistical Press (in Chinesisch).

SiU, Y.M. \& S.M. LI (1993): Population mobility in the 1980s: China on the road to an open society. - In: Cheng, J.Y.S. \& M. Brosseau (Hrsg.): China Review 1993. - Hongkong.

Solinger, D. (1985): Temporary residence certificate regulations in Wuhan. - In: China Quarterly 101: 98-103.

State Statistical Bureau (1991): Zhongguo 1990 Nian Renkou Pucha 10\% Chouyang Ziliao (Tabellierte 10\%-Stichprobe der Volkszählung 1990 der VR China). - Peking: Chinese Statistical Press (in Chinesisch).

Sumner, C. (1982): Crime, justice and underdevelopment: beyond modernisation theory. - In: SUMNER, C. (Hrsg.): Crime, justice and underdevelopment. London.

TAN, K.C. (1993): Urban-rural segregation in China. In: Geography Research Forum 13: 71-83.

TaubmanN, W. (1993): Socio-economic development and rural-urban migration in China since the beginning of the 1980s. - In: Tan, K.C., TaubmanN W. \& S.Z. YE (Hrsg.): Urban development in China and South East Asia. - = Bremer Beiträge zur Geographie und Raumplanung 25, Bremen: 163-185.

Taubmann, W. \& J. Fan (2000): Migrant enclaves in Chinese large cities. - Vortrag auf dem 29. International Geographical Congress in Seoul.

Todaro, M. (1989): Economic development in the Third World. - New York: Longman.

WU, C.T. \& X.Q. XU (1990): Economic reform and rural to urban migration. - In: LiNGE, G.J.R. \& D.K. Forbes (Hrsg.): China's spatial economy. - Hongkong. Wu, W.Y. \& X.Y. Zhang (1984): Zhongguo Renkou
Dili (Bevölkerungsgeographie Chinas). - Shanghai: East China Normal University Press (in Chinesisch). Xu, M.F. (1989): Cong Renkou Liudong Kan Huji Guanli Tizhi De Gaige Qushi (Bevölkerungswanderungen und Reformtendenzen im Haushaltsmeldewesen). - In: Shehui Kexue 2:36-40 (in Chinesisch).

XU, X.Q. \& Y. LI (1989): A preliminary study on the temporary migration and development of Guangzhou. - Vortrag auf der International Conference on Environment and Spatial Development of the Pearl River Delta am Department of Geography and Geology der University of Hong Kong.

\section{Zusammenfassung: Immigration, neue Armut und Segregation in Peking}

Dieser Artikel untersucht die aktuellen sozialen und planerischen Probleme infolge von Binnenwanderungen, speziell der starken Migration in die chinesischen Metropolen. Am Beispiel einer Stichprobenerhebung aus Peking gibt er einen Einblick in die Sozialstruktur der sogenannten floating population in der chinesischen Hauptstadt. Das Hauptinteresse gilt den Ursachen und Formen der städtischen Armut und Segregation. Diese werden im allgemeineren Zusammenhang der neuen Armut in den Städten auch anderenorts und im Zusammenhang mit neuen Strategien der Bevölkerungs-, Wirtschafts- und Stadtentwicklung in China gesehen. Es wird argumentiert, dass Peking und andere große Zentren Chinas den demographischen Veränderungen durch die gegenwärtigen sowie die zu erwartenden Zuwandererströme nicht gewachsen sind. Dies stellt auch eine Herausforderung an die Entscheidungsträger der Planung dar, ein neues Denken in der Stadt- und Regionalpolitik zu entwikkeln.

\section{Summary: Immigration, New Urban Poverty and Segregation in Beijing}

This paper sets out to review the current social and planning problems generated by population migration, especially the migration to the metropolitan regions in China. The analysis of a sample survey taken in Beijing provides insight into the socio-economic fabric of Beijing's so-called floating population. The causes and characteristics of urban poverty and segregation are examined in detail. They are seen in the broader context of new urban poverty in other cities as well as in the context of new development strategies for population movement and urban development for China. In view of the current and anticipated volume of inflow of migrants, this paper argues that Beijing and other big metropolises in China are not prepared for resulting demographic changes. The study calls upon decision-makers to develop a new paradigm in urban and regional governance. 


\section{Résumé: L'immigration, nouvelle pauvreté et ségrégation à Pékin}

Le présent article analyse les problèmes sociaux et de planification induits actuellement par les migrations urbaines internes, et tout spécialement par les forts courants migratoires propres aux métropoles chinoises. Il s'appuie sur un sondage à Pékin et donne un aperçu sur la structure sociale de la «floating population», la population flottante de la capitale chinoise. L'intérêt principal de cette étude consiste à faire ressortir les causes et les formes de la pauvreté et de la ségrégation urbaines. Celles-ci sont observées en relation plus générale avec la nouvelle pauvreté qui se manifeste aussi dans d'autres villes chinoises, en rapport avec les nouvelles stratégies de développement démographique, économique et urbain mises en place dans le pays. On fait remarquer que Pékin et d'autres centres importants de la Chine ne peuvent pas faire face aux transformations démographiques opérées par les flux d'immigration actuels et à venir. Ce constat interroge les décideurs de la planification et les pousse à déployer de nouvelles réflexions sur la politique urbaine et régionale.

Prof. Dr. Roger C.K. Chan, Centre of Urban Planning and Environmental Management, The University of Hong Kong, Pokfulam Road, Hongkong.

e-mail:hrxucck@hkucc.hku.hk

Prof. Dr. Chaolin Gu, Department of Urban and Resources Sciences, Nanjing University, Nanjing, Jiangsu, VR China.

e-mail:Gucl@nju.edu.cn

Dr. Werner Breitung, University of Macau, Faculty of Social Sciences and Humanities, P.O. Box 3001, Taipa, Macau.

e-mail: breitung@hotmail.com 